\title{
Kolmogorov Flow: Seven Decades of History
}

\section{Emmanouil D. Fylladitakis}

Electrical \& Electronic Engineering Department, Faculty of Engineering, University of West Attica, Athens, Greece

Email: e.fylladitakis@admie.gr

How to cite this paper: Fylladitakis, E.D. (2018) Kolmogorov Flow: Seven Decades of History. Journal of Applied Mathematics and Physics, 6, 2227-2263.

https://doi.org/10.4236/jamp.2018.611187

Received: October 25, 2018

Accepted: November 16, 2018

Published: November 19, 2018

Copyright (c) 2018 by author and Scientific Research Publishing Inc. This work is licensed under the Creative Commons Attribution International License (CC BY 4.0).

http://creativecommons.org/licenses/by/4.0/

\begin{abstract}
The Kolmogorov flow (k-flow) is generated by a stationary sinusoidal force that varies in space. This flow is rather academic since generating such a periodic forcing in an unbounded flow is difficult to appear in nature. Nevertheless, it allows for simple experimental measurements and for a fairly detailed analytical treatment. Although simple, the k-flow makes a good test case for investigating simultaneously inhomogeneous, sheared, and anisotropic features in a flow, and several studies concerning the stability, transition, and turbulence of the k-flow have been published. The present article reviews the most important published works incorporating the k-flow as a test-bed for studying fluid mechanics, testing numerical or experimental methods, or even studying the properties of the k-flow itself.
\end{abstract}

\section{Keywords}

Kolmogorov Flow, Magnetohydrodynamics, Two-Dimensional Flows, Review

\section{Introduction}

Near the end of the 1950s, A.N. Kolmogorov shifted his focus towards the study of the two-dimensional incompressible flows using a specific high wavenumber forcing and created the so-called Kolmogorov flow (k-flow). The Kolmogorov flow can be defined as a two dimensional and unidirectional shear flow with a specific sinusoidal mean velocity profile $(U=\sin z)$, which should always be maintained by any form of external forcing inside a viscous fluid. In $U=\sin z, Z$ stands for the cross-stream coordinate. Using $U=\sin z$, Kolmogorov's basic intent was to study and understand the transitions and complexities of turbulence in addition to the energy cascading process.

In most of the studies on turbulence, the meaning and intent of Kolmogorov's ideas are widely recognized. Since his pioneering ideas on locally isotropic tur- 
bulence, a lot of studies have been published on this specific problem. Moreover, a number of measurements have also been made in terms of assessing the characteristics of turbulence while using different forms of the natural media including the atmosphere, large wind tunnels, the ocean, etc. [1] [2]. These measurements have been effective in confirming the different predictions of the multidimensional theory that was proposed by Kolmogorov [3]. Since then, the $\mathrm{k}$-flow has been attracting a lot of attention and considerable progress and advancements have been made, both in the experimental and the theoretical aspects of the flow. Referring to the review article presented by Obukhov [4] and the studies conducted by Meshalkin and Sinai [5], it can be said that the k-flow belongs to a more diverse class of large-scale fluid instabilities. The Kolmogorov flow is more often defined as a form of sinusoidal flow whether the fluid being investigated is viscous or not. It is because of its simplicity, effectiveness, and accessibility in terms of analysis that Kolmogorov termed this flow optimal for being investigated in either theoretical or laboratory settings. The flow is also appropriate for conducting an investigation on fluid instability together with the transition towards turbulence [6]. Furthermore, different electrolytic fluids, soap films and other materials have been helpful in offering experimental measurements and realizations of this flow [3]. The k-flow has also been investigated and studied extensively in the field of magnetohydrodynamics (MHD) because suitably placed electric and magnetic fields can reproduce the k-flow fairly easy. It is used for the study of the varying dynamics of all types of electrically conductive fluids, electrolytes, different liquid metals, and plasmas. MHD turbulence and turbulent k-flow share common dynamical features, like the quasi-2D basic flow pattern and the inverse kinetic energy cascade and, thus, the k-flow has offered the test-bed for studies on fluid dynamics and flows in the field of MHD [7].

\section{Studying Fluid Mechanics \& Turbulence}

\subsection{Characterization of Turbulent Flows}

Surely turbulence is a most complex concepts and phenomenon, due to which it has been the subject of a large number of studies. It can be said that there is a lot more that needs to be done for grasping the complexities of turbulence. Researchers reaffirm that the study of turbulence is not easy because it demands a firm grip on the concepts of mathematics and physics. Despite the endless number of hypotheses that have been proposed in this regard, only a few of them have been able to generate definite predictions in terms of the ubiquitous nature of turbulence. It is also evident that there is no single universal theory of turbulence that has managed to provide accurate and deterministic predictions and applications of turbulence. Thus, it can be said that the true nature, cause, and mechanisms of turbulence need to be assessed and explored for shaping the future of fluid dynamics. In the first few decades of the 20th century, Richardson [8] and Kolmogorov [6] formulated a theory of turbulence using the concept of 
energy cascade. The kinetic energy is dissipated at a high constant rate from the large-size eddies towards the small eddies until the point at which the viscous action effectively dissipates the kinetic energy (KE). For the different incompressible Newtonian fluids, the following formula can be used for understanding the kinetic energy dissipation [9]:

$$
\varepsilon=2 v\left(S_{i j} S_{i j}\right)
$$

where $S_{i j}$ is the rate of the strain tensor and $v$ is the kinematic viscosity coefficient.

After having performed an analysis of the Navier-Stokes equations, it can be said that there are a number of rigorous limits on the rate of energy dissipation for variable incompressible flows [9]. It has also been demonstrated that in the case of unbounded turbulence, the rate of energy dissipation is influenced by the shape of the forcing. The upper bound approach method that was adopted from the in depth analysis of Doering \& Constantin [9] [10] revealed a dependence on a number of variable k-flow.

Firstly, all turbulent flows happen to be highly irregular. This is the reason for which most of the turbulence problems are dealt and studied in a statistical manner. Furthermore, turbulent flows are highly chaotic. However, it is important to consider here that not all chaotic flows can be classified as turbulent. There are always readily available supplies of energy that lead to an increased and rapid homogenization of the variable fluid mixtures. Turbulent flows are marked for having an intense and strong three-dimensional vortex generation principle or mechanism. This phenomenon is commonly referred to as vortex stretching. In order to sustain the turbulent flow, there is always a need of having a persistent supply of energy. The main reason is that turbulence always dissipates in an agile manner, as the resulting kinetic energy is continuously converted into internal energy.

Turbulent fluid flow has always been classified as one of the most complex and fundamental problems in physics. Additionally, it also holds great importance on predictions regarding heat transfer, the weather, ocean currents, etc. In the field of fluid dynamics, turbulence is defined as a flow regime marked by a number of chaotic changes and variations in properties. It includes a lower momentum diffusion, rapid variations in pressure and momentum [3]. In the case of the turbulent flows, a number of unsteady vortices seem to appear on the different scales while interacting with each other. At the same time, both the structure and the location of the boundary layer change, leading to a lower or reduced overall drag. In order to understand turbulent flows in two-dimensional and three-dimensional k-flow regimes, the aforementioned features need to be evaluated [11]. Thus, turbulence is the most complicated unsolved problem in the field of classical physics [12].

Kolmogorov's seminal studies and papers can be seen as empirical departures from the different scaling predictions that he presented. Thus, it is now confirmed that the turbulent scales cannot be termed self-similar and that they 
gradually become more intermittent as the size of the scale decreases. The characterization and visualization of these deviations are yet another contribution of Kolmogorov. The modern theories and explanations on fluid turbulence can be seen as going beyond the Kolmogorov Theory. During the past 50 years, a number of sophisticated and theoretical descriptions regarding fluid turbulence were presented; these also include the seminal contributions of Robert Kraichnan [13] [14] [15]. However, a number of researchers agree that his works on statistical field theory are based on the assumptions and insights offered by Nikolai Kolmogorov [16].

There have been a wealth of publications from researchers that attempted to characterize k-flow and turbulence during the last century. Most of this research can be summarized into recently published review papers. Two review papers initially presented at the Royal Society summarize a large part of the progress that had been made on the understanding of turbulence up to that point of time [17]. Frisch [18] summarized the progress that had been made on the scaling in fully developed turbulence, discussing the work of those that focused their research on this matter and noting that even though turbulence remains an unsolved problem, the actual problem is that there is no consensus on how the problem of turbulence should be formulated. The review of Hunt and Vassilicos [19] was focused on the research that has been performed on small-scale turbulence until that point of time. After summarizing all the research that has been done on the topic, the authors presented some extensions and applications based on Kolmogorov's hypotheses. They pointed out that the most widespread practical application of Kolmogorov's model has been for calculating the effects of turbulence, yet there is a very long list of other possible applications, such as sound production, transmission of light, mixing of species and fluctuating forces.

A third review paper was presented in the same publication [17] by Bray and Cant [20], summarizing the advancements that the research on Kolmogorov's turbulence had offered in the field of combustion. Bray and Cant summarized the research that had been performed with the particular application in mind, focusing on the insight gained by research that performed direct numerical simulations. They also identified the unresolved problems and, based on the data from previous numerical simulations, a new theoretical model for the mean flame stretch factor was developed. A more recent attempt aiming to improve our understanding of turbulence has been performed in 2014 by Suri et al. [21]. Via simulations using a 2D model and experimental realization of a quasi-two-dimensional flow, the authors derived an equation for the vertical profile of the horizontal velocity field for Kolmogorov-like and unidirectional flows. The effects of viscosity, magnetic field and layer thickness on the coefficients of the equation are also being discussed.

\subsection{Kolmogorov's Studies on Turbulence}

To begin with, the two-thirds law should be analyzed in order to develop a better 
understanding of the theory. The law states that the mean square difference of the different velocities along two points in turbulent flow is inherently proportional to the specific distance between the two observational points raised in the power $2 / 3$ in the area or regions of the different intermediate scales. With the modern development and formulation of new and more sophisticated measurement techniques, it is possible to gain new insights about the subtle structures and components of turbulence. Kolmogorov is also remembered for his contribution of giving a theoretical estimate in relation to the corresponding scale [22].

Kolmogorov was primarily concerned with the fluid mechanics and other related problems with relevance to the turbulent disturbances. These disturbances arise due to the hydrodynamic instability or inconsistencies in the flows of fluids that happen to have a small viscosity. Now here, the corresponding mathematical viewpoint or theory is highly complicated. In order to develop a better understanding of this phenomenon, Kolmogorov was of the view that a simple model should be used. He suggested that the model of the two-dimensional movement or motion of any viscous fluid caused by a periodic external source or force field can be used in this regard. And effective solution in terms of the problem of stability of the k-flow was presented by Lilly [23]. The model which was put forward was termed a convenient benchmark or object for furthering the theoretical investigations. No one had thought at that time that the model might be realized and used physically under the varying laboratory conditions. A number of other studies and references will be quoted later in order to offer a detailed idea about the k-flow and the mathematics behind its functionality [3].

The theories and works of Kolmogorov can be classified as the primary benchmarks for studying various natural phenomena such as turbulence. More specifically, Kolmogorov formulated a number of basic principles explaining the local structure of developed and complex turbulent flows. Moreover, these principles and concepts are based on a specific cascade model encompassing a large number of levels. There is also a sequence of scales ranging from large to small size. The large scales can be compared with the characteristic size of the entire system while the small scales can be seen as related to the order of the internal scale [24]. Making use of the available experimental data, Kolmogorov drew interesting conclusions: 1) all laminar solutions become unstable with the decrease in viscosity, and 2) with the viscosity approaching zero, the inherent smoothness of the observed solutions tends to decline in a very strong manner. It is important to note that the order of energy dissipation is determined based on the characteristic velocity as well as length, but is totally independent of the viscosity [24].

Based on these different conclusions, it was proposed that a turbulent solution is always present at low viscosity. In order to examine the problem in a more detailed manner, Kolmogorov proposed a simplistic model. It was referred to as the two-dimensional viscous flow that was caused by an external periodic force. The problems related to the stability of the flows were solved using the same ap- 
proach. Moreover, it was shown that the observed laminar flow happened to be unstable in relation to the long wavelength perturbations and disturbances [24]. However, there was not any kind of turbulent flow regime to be found. In short, a number of studies asserted that no turbulent flow regime could be obtained in two-dimensional cases. In order to understand turbulence and the various flow regimes, a third coordinate should be used. It has also been reported that the mechanism responsible for the onset of turbulence is marked for having a three-dimensional nature. Moreover, turbulence can also be studied using the numerical simulation of $\mathrm{k}$-flow inside a compressible shear layer [24]. Comprehending the fully matured or developed turbulence is important for a number of applications of geophysical flows. The k-flow has long been studied in the domains of geophysical fluid dynamics in relevance to the finite amplitude Rossby waves with the atmosphere as the setting [1] [2].

One of the primary notions of turbulence is that any turbulent flow is always composed of a number of eddies of varying sizes. These sizes help in defining the characteristic length scales for all the different eddies which in turn are characterized by velocity and time scales. In this case, the large size eddies are always unstable and eventually break up into a number of smaller eddies. At the same time, the kinetic energy of the large eddy is eventually distributed into several smaller sized eddies. These smaller eddies also go through the same process resulting in even smaller eddies. In this manner, the energy is passed down from the large to the small scales until reaching the point at which the kinetic energy is dissipated into internal energy due to the viscous action of the fluid [11]. It is clear that a turbulent flow is unique for having a hierarchy of different scales of different sizes due to which an energy cascade is formed. The dissipation of the kinetic energy scales with an order of Kolmogorov length $(\eta)$. Moreover, it is also important to note that the energy input for the cascade occurs due to the decay of the large size scales having an order L. The large scales can also differ from each other due to the order of their magnitude at relatively high Reynolds numbers. There are also a number of scales that are formed in between [25]. These scales happen to be very large in comparison to the Kolmogorov length, but smaller in comparison to the large scale of the flow. The mechanism and functions of these scales and turbulence have been explained in a number of studies and hypotheses proposed by Kolmogorov [6].

In his original works, Kolmogorov clearly postulated that for high Reynolds numbers, all small-scale turbulent flows and motions could be seen as statistically isotropic (meaning that no spatial direction or point can be discerned). However, not all of the large size scales in a flow can be termed isotropic primarily because they are influenced and determined on the basis of the specific geometrical features and characteristics of the boundaries. Yet another main idea of Kolmogorov was that this form of geometrical as well as directional information is decreased or lost inside Richardson's energy cascade. At the same time, the scale is also decreased or reduced so that the inherent statistics of the different small scales might come to have a universal character. In other words, the statis- 
tics for all small scales are the same when the Reynolds number is effectively high [6]. These findings were the source of a number of theoretical studies that were focused on the derivation of Reynolds number for accessing the onset of the fluid instability in different forms of unstrained k-flows. These studies also extended into unstable regimes using various forms of numerical simulations [3]. The results indicate that different small-scale instabilities result in a negative viscosity that in turn seeds a cascade of energy in relation to the injection scale. A number of researchers agree that the insights offered by Kolmogorov were useful in providing simplistic visualizations of the ingredients involved in two-dimensional turbulence. Kolmogorov further assumed that this cascading process occurs in a remarkably self-similar manner [7]. In other words, the eddies that share a given size behave in the same or similar manner in comparison with the ones having a different size. This assumption, combined with the $4 / 5$ law, helped in formulating general scaling predictions that are used in the numerical simulations of turbulence [3].

Different modifications of the Kolmogorov model have also been used in the past few decades. It is asserted that in order to have a more precise description of the different thin layers inside a fluid, a modified version of the k-flow can be used. These different forms of the k-flow also take into account the external friction, which is the friction at the wall. It should be considered here that the external friction is extremely crucial for having a correct estimate of the Reynolds number, which is of the order 103. Various versions and modifications of the $\mathrm{k}$-flow, like the generation of different 2D turbulence, large-scale formations of these disturbances and all forms of vortex merging have been extensively applied in laboratory and natural settings. In addition, Kolmogorov's theories and works have also been applied in the simulation of hydrodynamic flows [16].

\section{Recent Studies on the Properties of the K-Flow}

\subsection{Stability \& Bifurcation}

\subsubsection{The Early Experimental Background}

The two-dimensional flows are considered powerful tools for conducting a theoretical study on the transition of turbulence. These forms of theoretical investigations demand less analytic and computational power in relevance to the three-dimensional flows and also allow for the creation of different forms of stationary two-dimensional flows using the principles and concepts of magnetohydrodynamics. At the same time, it is also critical to consider that spatially periodic flows play an important role in terms of two-dimensional flows primarily due to their high levels of degrees of symmetry.

There are three classes (or types) of these flows that have distinct and unique symmetry properties. As the instability of these spatially periodic flows happens to be a major theoretical problem, there is a need for conducting detailed and comprehensive investigations of the phenomenon. One of the first theoretical studies in this regard was conducted by Meshalkin and Sinai [5]. Most of the studies in this regard are based on the ideal assumption that is based on an un- 
bounded fluid. Furthermore, the governing mechanism behind these studies is the Navier-Stokes equation. The parallel flows were also reported to be unstable against the various perturbations that used a very large scale in comparison to the periodicity length and other related features of the basic flow. In addition, this form of instability, which is also referred to as the negative viscosity instability, has also been found in a number of rhombic as well as square eddy lattices. However, no form of large-scale instability was detected in terms of triangular vortices. Instead, there was a form of oscillation reported for a specific form of vortex lattices. For about ten years now, the studies conducted on the topic in the field of magnetohydrodynamics have empowered us to generate periodic two-dimensional flows in all forms of laboratory experiments. One of the first successful attempts in this regard was made by Bondarenko et al. [26]. They observed the k-flow inside a specific electrically conducting fluid that was driven by an electromagnetic field. The indications and results of the studies conducted on square and triangular vortex arrays have been unable to conform to the different theoretical predictions [27].

At the same time, the specific Reynolds number in addition to the predicted large-scale instability was not observed. It was also reported by Bondarenko and a number of other researchers that the friction inside the layers of fluids is highly crucial for the instability and for comprehending the dynamics of the fluid flow [26]. Moreover, the observed instabilities that were observed were dependent heavily on the varying number of spatial periods. In other words, these instabilities were influenced by the degree of confinement present in the system. Despite an increasing number of studies that were focused on the confinement and wall friction factor, a detailed and comprehensive quantitative approach was always lacking [27].

\subsubsection{Stability Analysis \& Drag Reduction}

Few of the many early studies on the hydrodynamics of non-Newtonian fluids contained analytic results and, even then, the results were obtained only for exceedingly simplified problems. In the early 1990's, Brutyan and Krapivskii [28] published one of the first studies to provide a thorough analysis and investigated the stability of k-flow in an incompressible viscoelastic fluid. The authors mathematically determined the critical Reynolds number and obtained the first known analytic result in the theory of stability of non-Newtonian liquids at the time of their study. Around the same period, Andr'e Thess [29] also performed an extensive study of the instabilities in two-dimensional spatially periodic flows. His work was divided into three published parts; in part I Thess examined the linear stability of parallel two-dimensional flows (i.e. Kolmogorov flow). In part II, he modifies his study to arrays of vortices with square symmetry [30]. Finally, in part III he further extended his study to a third symmetry, assuming triangular alternating vortices [31]. Through these papers, Thess proposed a reconsideration of the linear stability of two-dimensional periodic flows in light of experimental results. For Kolmogorov flows, Thess calculated the precise critical 
values of parameters at which instability sets-in for both bounded and unbounded flows, which he then tried to verify experimentally. The experimental results, however, demonstrated that the instability threshold is strongly overestimated, suggesting that this was due to magnetohydrodynamic parameters discussed in [32] and [33], but the wave number was in good agreement with the study's theoretical results. In the second part of his work, Thess attempted to bridge the gap between theoretical and experimental studies via three major methods paying attention to the case of high/infinite Reynolds numbers, studying the influence of lateral confinement and revealing the spatial structure of unstable modes. Finally, in the third part of his study, Thess extended his prior studies by investigating an inviscid flow with hexagonal symmetry. Although the study displayed a good replication of previous experimental results in electromagnetically driven flows, the author suggests that other specific aspects, including magnetohydrodynamical, would have to be considered in relevant studies. In another paper published soon afterward, Thess examined the inviscid instabilities in two-dimensional periodic flows [34]. Although largely based on the previous publications, this paper presented new theoretical results on the stability of non-parallel flows driven by a Lorentz force, displaying that waves propagating along the symmetry directions occur in the triangular lattice. The results were in good agreement with previous experimental studies, developing a theory that could reproduce the experimental values for the instability threshold in a triangular lattice.

Dubrulle and Frisch also published their findings on flow instability in the early 1990's. In their work, the authors embraced a general formalism to determine eddy viscosities for the incompressible flow of arbitrary dimensionality subject to periodic forcing in space and time [35]. A section of their manuscript is devoted to layered flow, with detailed results for time-independent parallel flows, including variants of the k-flow. However, the authors noted that flow regimes presenting negative viscosity instabilities cannot be examined using the restricted framework assumed for this study, noting that a correct theory should also include dissipative and nonlinear terms. In a companion paper, however, Henon and Scholl used the numerical observations of Dubrulle and Frisch and, performing simulations made with a lattice-gas algorithm, predicted a non-transverse instability for a modified k-flow [36].

Several years later, Frisch et al. [37] published their work regarding large-scale dynamics of the k-flow near its threshold of instability in the presence of the beta effect (Rossby waves). The paper was centered on a single-dimensional "toy model" for studying an instance of the interaction of turbulence and waves. The paper is divided between results specific to the $\beta$-Cahn-Hilliard equation [38] and results that may apply to a broad class of problems involving resonant wave interactions.

Wang et al. [39] studied turbulence as well, although their work took a different, quantitative approach. The fundamental hypotheses underlying Kolmogo- 
rov-Obukhov turbulence theory, also known as the "K62" theory, were quantitatively examined [40]. The authors performed direct Navier-Stokes simulations (DNS) at 5123 resolution with Taylor microscale Reynolds number up to 195. Three very different types of flow were considered: free-decaying turbulence, stationary turbulence forced at a few large scales, and a 2563 large-eddy simulation (LES) flow field. Both the forced DNS and LES flow fields showed realistic inertial-subrange dynamics. While their results were limited to moderate turbulence Reynolds numbers, the authors advised the readers not to draw definite conclusions based on the DNS results available at the time and noted that their results were supportive evidence of the K62 theory. Three years later, the authors published a paper on the same subject [41]. Using direct numerical simulations (DNS) and large-eddy simulations (LES) of velocity and passive scalar in isotropic turbulence (up to 5123 grid points), they quantitatively examined the refined similarity hypotheses as applied to passive scalar fields (RSHP) with Prandtl number of order one. For the first time, the exact energy and scalar dissipation rated have been used and scaling exponents were quantified as a function of the local Reynolds number. Their study demonstrated that the velocity increments depend on the locally averaged dissipation rate and enstrophy, while the scalar increments depend on the local average dissipation rates alone. The results of the study compared well with those of other numerical and experimental studies. The authors specifically mention an interesting outcome of their study; the fact that the small-scale features of the scalar field and those of the velocity field share both differences and similarities.

Another study concerning the stability of k-flow investigated the critical Reynolds number while examining the instability of the flow in soap films [42]. The study declares that the idealized theoretical model, which is based on a linear stability analysis, predicts a critical Reynolds number nearly fifty times lower than the critical value derived from a soap film experiment. The study suggested a model with two-dimensional motion equations that provides better agreement with the experimental results than previously suggested models; however, as stated by the authors, the model still has inadequacies and only a full three-dimensional analysis of the system would fully describe the actual three-fluid flow mechanism.

The viscous dissipation also is an important aspect of turbulent flow and, therefore, the bounds associated with it are of great importance. The elementary bounds on various aspects of the viscous dissipation rate in Navier-Stokes flow with Kolmogorov forcing have been examined in the early 2000's by Childress et al. [43]. The study revealed that the bounds are rather generic and, thus, any improvements must capture key features that typify the turbulent flow response.

In 2002, Chen and Price [44] performed an instability analysis of liquid-metal Kolmogorov flow in a straight duct. After a thorough analysis of the proposed mathematical theorem, the authors performed numerical experiments to determine the instability thresholds of metal fluid flow. The results of this analysis were a good match with those of Thess [29] and Kolesnikov [45]. Oparina and 
Troshkin [46] performed an analytical study to determine the stability of the $\mathrm{k}$-flow in a channel with rigid walls. Their study displays that $\mathrm{k}$-flows with short periods will remain stable inside a channel with rigid walls. The study was based on theorems that describe the bifurcation of the solution into a new flow regime, either steady-state or self-oscillating, when the Reynolds number attains a certain critical value. A few years later, in 2005, the authors also published a paper on the secondary electromagnetically driven flows [47]. The main motivation behind the second paper was to show the qualitative differences between elementary $(N=1)$ and extended $(N>1)$ wall-bounded flows. Based on similar numerical experiments as those of their previous work, the authors offered insight on the predicted development of secondary flows in a duct, concluding with a numerical model that can approximate such flows in bifurcating steady-state solutions.

The instability observed in the k-flow has also managed to generate a large interest in the domain of mathematics and other related fields. This flow is able to show a large-scale instability in terms of the negative viscosity type. For most of the super critical conditions, it is important to note that the inverse cascade happens to be a distinctive feature of for the different two-dimensional flows. Initially, we should consider the two-dimensional flow of any incompressible fluid that is being governed by a specific dimensionless equation. The mathematical treatment for the instability equation is determined by taking into account the type of system i.e. whether it is bounded or not. It is also expressed on the basis of some proper boundary conditions [27]. It is because of this behavior that the small-scale forcing can be termed an effective mechanism or means of generating a large-scale two-dimensional turbulence. It should also be noted that the linear stability in relevance to large-scale perturbations has also been investigated by making use of multiple scale analysis. Flow instabilities have remained a classical subject in the field of fluid dynamics. Moreover, the theoretical studies about their presence and occurrence in most polymer solutions are of paramount importance in a number of industrial applications. A satisfactory understanding of these flows involves a consideration of the viscoelastic behavior of these fluids, which is the main reason why the subject of stability in relation to $\mathrm{k}$-flow has been investigated in a number of studies and experiments [27]. When considering the linear stability of the parallel k-flow, it is important to consider the viscosity, the confinement, and the linear friction. These computations help in the provision of different neutral instability curves in terms of the parameter space together with the wave numbers and speeds. A great deal of evidence suggests that all stability parameters are dependent on confinement in a non-uniform manner. It has also been shown that all forms of weak transverse confinement decrease the resulting longitudinal wavelength of the perturbations at the onset of the instability. Moreover, strong confinement helps in changing the character or nature of the instability into the one having an oscillatory instead of an exponential nature [27].

Boffetta et al. [27] also performed numerical simulations to determine the 
drag reduction in turbulent k-flow [27]. Using a linear viscoelastic model, the authors examined the three-dimensional turbulent k-flow and demonstrated that drag reduction does take place above a critical Reynolds number. In their study, an expression for the dependence of the critical Reynolds number on polymer elasticity and diffusivity was proposed. They concluded that the drag coefficient can be expressed as a function of the rescaled Reynolds number only, that this function is universal with respect to the fluid characteristics, and that its shape can be derived by simple phenomenological arguments. However, the numerical verification of these expressions and conclusions using nonlinear models has not been addressed in their work.

The nonlinear dynamics of viscoelastic k-flow have been examined by Bistagnino et al. [48], both analytically and numerically. The authors specifically noted that the physical reason for their study was that, even though there are no physical boundaries, this flow has several analogies with channel flows and is one of the few known solutions of the Oldroyd-B model [49]. The study concludes that the weakly nonlinear dynamics are described by equations that resemble those introduced by Cahn-Hilliard [38]. However, the equations contain a fifth-order nonlinearity and with coefficients that depend on the Deborah number. They also performed a study on drag reduction, displaying that the injection of polymers induces an increase of the mean flow and reduces the drag coefficient. The main qualitative conclusion of their study is that drag reduction appears to be a phenomenon coupling large and small scales. Another study based on numerical simulations that investigated the dynamics of the two-dimensional periodic $\mathrm{k}$-flow of a viscoelastic fluid, described by the Oldroyd-B model, has been presented two years later by Berti and Boffetta [50]. The authors investigated the destabilization of the k-flow induced by the elastic forces associated with the dynamics of polymer molecules in the solution. The study revealed that above a critical Weissenberg number (Wic $\approx 10$ ), a transition to new dynamical states was observed. The authors noted also that the establishment of mixing features is possible in that state.

Mishra et al. [51] recently performed numerical simulations on two-dimensional Kolmogorov flows, studying and identifying all of the possible flow regimes and their bifurcations, yet focusing on the reversal and condensate regimes. The parametric study was mainly performed for a varying $\mathrm{Rh}$, which represents the ratio of the inertial and viscous terms, revealing that its increase would render the flow unstable, following a series of transitions thoroughly described in the study. The results of this study were in good agreement with both other similar numerical studies and with experimental observations. Finally, the study includes the analysis of the energy transfers among Fourier modes and displayed the symmetry of flow reversals in Kolmogorov flows.

\subsubsection{Stability of the Beta Plane Kolmogorov Flow}

The consequences and outcomes for the varying geophysical Beta effect will need to be explored. It can be shown that even in the limit $\beta \rightarrow 0$, the Reynolds 
number can be reduced in response to the generic effect of beta. The stability of the Beta plane of geophysical k-flows has been studied by a number of researchers and authors. Lorenz [52] and Gill [53] evaluated the stability of this flow at $\alpha$ $=0$ using an inviscid case. In terms of a viscous case, Frisch et al. [37] took a different value for the $a$. An analysis of these studies has revealed that different values of alpha have been used in this regard [54].

\subsubsection{Stability of an Oscillating Kolmogorov Flow}

For oscillating flows, there is a need for considering the time and traverse direction. This issue can be reduced to an infinite algebraic problem. By using a number of recurring fractions, it can be proved that the time-independent flows are quite unstable to the different perturbation modes. It should be noted here that these modes do not possess the periodicity of the basic flows when considered in traverse directions. Instability has also been identified for a number of inviscid cases even when the different perturbation modes had the same periodicity [55].

It is important to consider that the stability of k-flow has been studied using a number of different materials and fluids. A number of studies have been carried out using the ordinary viscosity and the lateral walls. In terms of the strongly confined systems encompassing only a single period of the k-flow, a form of oscillatory instability was identified. On the other hand, the instability of the flow without any boundaries has also been evaluated using various quasi-periodic perturbations. This specific viewpoint has helped in understanding the stability of the flow while using the confined systems. It can be said that the future studies should be dedicated to exploring these instabilities in more detail [56].

\subsubsection{Bifurcation Analysis}

The studies in this regard have shown that with the increasing strength of the variable force, the system exhibits a number of different forms of bifurcations in response to steady states. In addition, the traveling waves, torus solutions and all other related factors also come to influence these bifurcations. Different types of bifurcation analysis techniques have also been used for exploring the topic in detail [56].

In 1991, Platt et al. [57] performed a numerical investigation of chaotic $\mathrm{k}$-flows, focused on defining transition states for a range of the bifurcation parameter Reynolds number $(R e)$. The authors determined that there were two main regimes of flow, small and large-scale structures, with each corresponding to different ranges of Re, and described the sequence of bifurcations that takes place in each of these two flow regimes.

In his Ph.D. thesis, Philip Love [58] computed the bifurcation structure of Kolmogorov and Taylor-Vortex flows. The author used the numerical Recursive Projection Method to calculate many branches of both Kolmogorov and Taylor-Vortex flows. The results were validated via comparisons of steady state flow calculations to turbulence calculations. The author also concluded that turbulent 
flow originates from k-flow, through a series of bifurcations. By following bifurcating branches, the author observed the existence of worm-like structures, which may transform into the similar formations present in turbulent flow.

Legras et al. [59] published a perturbation theory, based on numerical simulations. Their work showed that the inverse cascade of kink/anti-kink annihilations, which are a characteristic of k-flow in the slightly supercritical Reynolds number regime, can be halted by the dispersive action of Ross by waves in the $\beta$-plane approximation. The main result of their theory was the following equation:

$$
|\beta|>\beta_{c}=\sqrt{35389440 \frac{\mathrm{e}^{-s \Lambda}}{\Lambda^{5}} s^{5} \lambda_{3}^{2} \frac{1}{4+9 q^{2}}}
$$

The equation gives the minimum value of $|\beta|$ capable of stabilizing a solution of period $\Lambda=L / N$, with $N>1$.

Several years later, the same authors also examined the large scale instabilities of generalized turbulent Kolmogorov flows [60]. The authors used the Clark-Smagorinsky model [11] to investigate instabilities in Kolmogorov flows for which the molecular viscosity has been replaced by a parametric eddy viscosity. Their analytical study concluded that such instability is obtained for parameterized large eddy simulation equations, as soon as the filtering length of the simulation is smaller than a threshold.

By examining the bifurcation curves of solutions to the k-flow, Matsuda and Miyatake [61] performed an analytic study and presented the mathematical formulas for the second derivatives of their components concerning the Reynolds number at bifurcation points. Their work mathematically proved that numerical computation is possible by choosing finite numbers of points.

The recent paper from Tithof et al. [62] presented a combined experimental and theoretical study on the instabilities of $\mathrm{k}$-flows meant to compare the validity of a numerical model to real-world results. This also is the first k-flow study to provide a quantitative analysis of the secondary instability that generates a time-dependent pattern of vortices. The authors performed physical experiments using electromagnetic forcing to drive a quasi-two-dimensional shear flow in a thin layer of electrolyte suspended on a thin lubricating layer of a dielectric fluid. Their theoretical study was based on the $2 \mathrm{D}$ model from one of their previous works [21] and, according to the authors, the numerical model predicts the modulated flow pattern with fairly reasonable accuracy. It was indicated, however, that the accuracy of the numerical predictions does decrease as the Reynolds number increases.

\subsection{Stratification and Heat Transfer}

On the subject of stratification and heat transfer, k-flows can be assessed and investigated by using a weakly stratified two-dimensional fluid flow. Firstly, the amplitude equations and measures for the whole system will need to be derived. Both high, as well as low Peclet numbers, will be used in this regard. To begin 
with, it is important to note that the stability of the viscous sheer flows is a comparatively difficult problem. For decades now, the problem has continued to interest and influence scientists and researchers from all over the world. Without taking into consideration the effects of stratification together with compressibility, the linear theoretical analysis of the problem might not be easy. It should be noted that this analysis is primarily based on providing the solution for the famous Orr-Sommerfeld equation. It is also important to consider that only a few generalized results and solutions have been obtained for this specific equation. As mentioned earlier, deriving the critical Reynolds number is crucial for understanding the occurrence of stratification and heat transfer in k-flows. More specifically, the resulting instability can be measured more effectively using different values of the Reynolds number. In an effort to grasp the nature and functions of stratification, a number of studies used this approach.

Stratification and heat transfer can be assessed using gravity. More appropriately, gravity is used in a direction that happens to be transverse to the k-flow. This strategy can be used to explore all forms of weak stratification. It can also be deployed for modifying the basic versions of linear instabilities in addition to the nonlinear development. As the stratification exerts a strong stabilization effect, an inverse cascade effect can be anticipated. Yet again, there are a number of geophysical motivations that play an important role in this regard. For this reason, it is also important to measure (and, in some ways, control) these motivations. One such motivation is the stability of the different vertical shear flows generated by the atmosphere [63]. Moreover, the internal gravity waves that possess finite amplitude can also be classified as one of these geophysical motivations. The phenomenon of stratification and heat transfer in k-flow has also been investigated in the laboratory settings. However, the direct application of these experiments will not be considered here. The focus is primarily to offer a description of the mechanical problem [1] [2].

In order to successfully formulate an assessment, it is imperative to start out with the factors of vorticity as well as heat equations for the two-dimensional stratified flows. This flow is defined using the $x$ - $Z$ plane while measuring the gravity along the $z$ coordinate. Secondly, it is crucial to exploit the incompressibility of the flow in order to express the different components of velocity in terms of the different stream functions. At the same time, the Kolmogorov shear flow that is present in the background is measured and characterized by $\Psi 0=$ $U 01 \cos (z=I)$, where $U 0$ is the amplitude. In the formulation and construction of the equations, it is important to keep a number of things in mind: 1 . the equations are dimensionless, and 2. these equations will be used for recovering the unstratified k-flow [1]. The studies conducted by Balmforth and Young [1] have confirmed that the introduction of varying forms of stratification can be helpful in suppressing the observed instabilities. The second instability in this regard happens to be a conductive one that operates primarily through the creation of a wide scale thermal diffusion. This specific instability is reported to arise with a much stronger form of stratification. It also leads to the generation of prominent 
staircases in relevance to the buoyancy field. The different steps seen in the staircase are marked for having their own nonlinear dynamics. These steps have also been reported to show coarsening during the various phases of the stratification process. In the second study conducted by Balmforth and Young [2], the available configurations and parameters were such that only viscous instability had been present due to which there was no evidence that could confirm the layering [1]. A further study based on [1] and [2] by Sarris et al. [64] examined the laminar convection flow in enclosures driven both by a nonuniform Lorentz force of Kolmogorov forcing type and by a buoyancy force. The researchers found that a proper combination of the magnetic and gravitational forces may enhance heat transfer up to $40 \%$ over the usual natural convection heat transfer rates.

The stratified shear flows also arise in a number of astrophysical fluids. One of the core issues in this context is realizing the manner in which the unsteady eddy motions result from the different forms of steady forcing. Furthermore, it is crucial to know how the motion is able to rearrange as well as transport all of the fluid properties. The k-flow has also been rationalized as a mechanism for understanding the unstratified flow dynamics and transition of turbulence. $\mathrm{Nu}$ merous laboratory experiments and studies have reported the generation of different staircases separated by sharp interfaces. In most of the laboratory settings, these staircases have been created using dragging bars or grids from inside the salt-stratified water [65]. The turbulent environment of the ocean and other related settings are believed to cause the same effect on the flows.

Thermal convection and other similar measures have also been used for gaining a deeper understanding of the heat transfer in combination with stratification [2]. Although the phenomenon has not been proved empirically, it has been assumed that the turbulent field is the primary ingredient for accessing the layering problem. In other words, the process of layering offers a number of insights about the stratification process. Based on the same premise, a number of mathematicians and researchers have tried to produce simple models for evaluating turbulent stratified flows. However, it should be noted that most of these models tend to rely on simplistic and empirical parameters of different forms of turbulent transport. The prevailing notion in this regard is that the point at where the flux decreases together with the gradient, the observed stratification is quite unstable. It has also been reported that the staircases can also be seen for lower values of the Reynolds number. An analysis of this process gives rise to a number of key questions for studying stratification in terms of k-flows. Moreover, it leads to the need for more detailed and analytical explorations using the principles and concepts of fluid mechanics [2].

The instabilities that are catalyzed by the different viscosities have also been measured and investigated in a number of other contexts. Researchers also report a temptation of rationalizing the prevailing instabilities as analogies. Furthermore, a number of different indications have been reported in current theories and principles that are being used for understanding stratification. A recent 
paper from Ponetti et al. [66] describes the transitions in a stratified Kolmogorov flow, with the researchers performing a numerical study to examine the transitions that lead the flow to chaotic states, identifying that the flow reaches chaotic configurations through two different routes: one involving drifting states and one involving a gluing bifurcation.

\section{Turbulent Mixing in Stratified Flows}

The range of the different mixing efficiencies and turbulent diffusion happens to be very large. For this reason, a proper parameterization of the geophysical flows is important. There are a number of experimental settings and procedures that have been used in this regard, such as grid-generated turbulence and air bubble turbulence. There are also a number of other studies in which basic instabilities have been investigated using density fluctuations and stratified flows. The mechanism through which the process is achieved is based on constantly changing the parameters. The effect of changing these parameters in an uncalculated manner affects the results negatively. For this reason, it is imperative that the process should be completed in a rather careful manner. Another important point to consider is the flow visualization that is integral in terms of stratification. Among the different fluctuation measurements, it is seen that there is a region with an intermediate $R i$ that allows for a higher and direct transport of the unmixed fluids and materials through the interface [67]. At the same time, experiments conducted using bubble generated mechanisms exhibit rather different forms of mixing efficiencies. An analysis of these studies and investigations has shown that a decrease in the different mixing efficiencies can be seen only for the stronger density interfaces. It can also be argued that for higher values of $R i$, a longer time span is needed. During this time, the interface is also able to offer support for the internal waves possessing higher frequency and buoyancy. Moreover, the mixing values and measures prove to be of much help in terms of analyzing the different parametric ranges [68].

It is also important to consider that turbulence and stratification are one of the primary features of environmental flows. It is due to their interactions that a number of flow phenomena have generated a lot of interest in the domain of fluid dynamics. There are several categories of the turbulent flows that should be classified and investigated for the purpose of understanding stratification, turbulence and heat transfer in a more effective manner [69]. In his review article, H. Fernando [69] presented a short review of turbulent mixing in stably stratified fluids. The study concludes that, up to the year that the manuscript was published, there was little consensus among researchers regarding various issues.

A recent study explored the formation of coherent structures in a weakly stratified Kolmogorov flow under the effect of minor linear drag [70]. The model used for this study was based on the core Kolmogorov model with the effects of linear vertical density stratification and drag taken into account. The bifurcation diagram was computed for a Reynolds number of up to 100 and the formation of three coherent structures was observed. The authors conclude that future studies 
using higher Reynolds number values are necessary to reveal the effects of stratification and drag on these coherent structures.

\subsection{Hydrodynamic Fluctuations in Kolmogorov Flow}

Bena et al. studied the hydrodynamic fluctuations in the k-flow in both the linear [71] and non-linear [72] regimes. Their work was based on Landau-Lifshitz [73] fluctuating hydrodynamics, mainly because, as the authors proclaim, of its relative simplicity. The main purpose of the articles was the study of the statistical properties of k-flow via numerical calculations. The first study [71], focusing on the linear regime, presented that the validity of the incompressibility assumption is flawed, leading to unsatisfactory results; however, the problem becomes too complex if compressible hydrodynamic equations are used, due to the boundary value problem. By basing their theory on the relative simplicity of the k-flow, the authors managed to display that, in the long time limit and for linearized fluctuating hydrodynamic equations, the flow behaves as an incompressible fluid irrespectively of the Reynolds number. In their second published paper [71], which explored the nonlinear regime, the authors verified that the incompressibility assumption leads to a wrong form of static correlation functions, except near the instability threshold. They used a perturbation technique to find the limits of where the macroscopic behavior of the fluid is not affected, displaying that the stochastic dynamics of the system is governed by two coupled nonlinear Langevin equations in Fourier space when the system is close to the instability threshold.

Mansour et al. also [74] performed particle simulations of the k-flow and analyzed them by the Landau-Lifshitz fluctuating hydrodynamics. The authors concluded that a spurious diffusion of the center of the mass has no effect on the average macroscopic behavior of the system, yet corrupts the statistical properties of the flow and is an issue for microscopic simulations. Their study provides an analytical expression for the corresponding diffusion coefficient. Several years later, a molecular dynamics simulation of spheres was performed in order to study the behavior of k-flows in granular matter [75]. The spheres interacted via elastic collisions and a force mimicking the effect of capillary bridges. It is noted that the instability of the flow is present even in dry granular matter, where particle interactions are limited to inelastic collisions.

The advection of passive particles in the k-flow has been studied by Beyer and Benkadda for two different regimes of the flow [76]. The regimes were cross-checked based on the same parameters used in Platt's study of chaotic k-flow [57]. Their study displays that the advection of particles is different within different regimes, even though the asymptotic diffusion remains normal in all cases. The authors concluded that time characteristics alone are inadequate to define anomalous transport, which requires both time and space characteristics to be simultaneously present. Mitchell and Grigoriev [77] presented a numerical study, investigating the change of the mixing properties associated with the transition from laminar to turbulent regime in a two-dimensional k-flow. The au- 
thors concluded that the mixing efficiency improves as the forcing is increased, i.e. steady flows are the worst mixers and turbulent flows the best. However, neither the complexity of the flow or the mixing efficiency increase monotonically. It was noted that the mixed area fraction of a class of time-periodic and quasi-periodic flows can be accurately described by a perturbative approach, although the flows considered in the study cannot be considered weakly perturbed.

\section{Recent Numerical and Experimental Research on Kolmogorov Flow}

A number of experimental studies and investigations have been carried out in order to understand the nature and functions of k-flow. We will begin this section by presenting the most widely accepted and used modeling and simulation methods, highlighting their primary advantages and limitations. The section will continue with the presentation of the experimental studies that have been performed on Kolmogorov flows, summarizing their purpose and outcomes.

\subsection{Modeling and Simulation Methods}

Mathematical and all other forms of modeling are useful methods for the analysis of complex systems. Modeling is also used at times when it is not feasible to conduct experiments with the real systems. Provided that models offer adequate descriptions of the different casual relationships, conducting experiments with them using computer aided mechanisms is one of the most effective and efficient measures. In terms of k-flows, there is a number of modeling and simulation techniques that can be used, including conceptual, functional, constraints, declarative, and multi-model designs [78].

\subsubsection{Conceptual Models}

Conceptual models are used for making systems that are easier to assess and use. However, the techniques that are used in terms of these models design might not be good enough to understand and grasp the complexities and difficulties of the k-flow. A detailed conceptualization process can formulate and evaluate the model. During the entire process, it is also expected that new principles and concepts will be formed. The techniques that are used in this regard have been primarily formalized for being used with the most simplistic models. One of the main advantages of the design is that it helps in formulating systems that are simple to use. However, it can also be taken as a disadvantage because the process limits the functions of the resulting systems [79].

\subsubsection{Declarative Models}

A model can be termed declarative if it is successful in determining and analyzing the actions of different agents and the manner in which these states can be changed. More specifically, these models are used for specifying the reactions to states. Moreover, these models allow for adding qualitative facets and considerations without any compromise on the accuracy. It can also be said that declara- 
tive modeling is based on developing a model in a diagrammatic manner. It should also be pondered that the term points out towards the use of a procedural approach. Moreover, the model is represented in the form of facts that are true. In other words, these facts are used for defining the model [79].

\subsubsection{Functional Models}

Functional models help to determine the mode of the functions and operations in the system. A functional model always contains a number of functional entities. It can also be defined as a way of defining the functional aspects and features of a system. It is due to the wide scale benefits and comprehensiveness of the model that it has been used constantly in a large number of engineering applications. One of the prime advantages of the design is that it records all of the important phases and segments of the modeling process. Moreover, the systems with a number of functionally designed segments and parts can be modified easily. At the same time, the functional modules can also be used more easily using this modeling approach [79].

\subsubsection{Constraint Models}

The different entities in declarative models are defined in terms of constraints that determine their nature as well as the relationship. The program code is preferably kept separate from the specific modeling description that has been used. In order to develop a model from the constraints, it is imperative to modify its description using the natural language. The success of the model is based on using the right constraints and modeling techniques [80].

\subsubsection{Multi-Models}

This form of modeling can be seen as an extension of the object-oriented designs and techniques. One of the major contributions of these modeling techniques and methodologies is that they allow for different forms of mapping between the real and digital worlds. Furthermore, this form of mapping allows for a more realistic view of the design. The process of generalization and aggregation is used in order to form a number of hierarchical structures. More specifically, these models are created using a number of constructing objects and then connecting them. It can be said that the resulting models share the advantages and benefits of the different modeling procedures and designs. It has also been observed that some levels might be functional or declarative, while others might be marked for possessing the features of other modeling designs [79].

One of the first numerical simulations related to k-flows has been performed to investigate the stochasticity properties of dynamical systems [81]. Although stochasticity is defined in a qualitative way, the proposed method allowed for the definition of a quantitative parameter, the "entropy-like quantity", which is related to the Kolmogorov entropy for associated flow.

\subsection{Simulation Techniques for Kolmogorov Flows}

The primary purpose of a simulation is to gather the maximum possible infor- 
mation about a system using the most convenient measures and strategies [79]. However, the use of different models and simulation techniques for understanding the Kolmogorov flows has been subject to a number of controversies. Although a wide range of studies and researches has been conducted in this regard, there are a number of drawbacks and complexities that need to be ameliorated.

Kalis and Kolesnikov [82] performed a numerical study of the k-flow in a strong magnetic field. The authors proposed that instead of two linear electrodes located perpendicular to the field, the linear electrodes should be positioned periodically along the $\mathrm{x}$ axis, through which a DC current $\mathrm{I}$ is periodically applied. It has been suggested that this approach allows for the formation of k-flow in a channel with non-conductive walls. In 1997, Posch and Hoover [83] suggested an alternative method for the simulation of hydrodynamic flows, which has been applied on a two-dimensional k-flow. The method was based on Smooth Particle Applied Mechanics and, thus, the authors baptized it SPAM. The two-dimensional k-flow that the method was applied on had the fluid at the top and bottom half of a tube accelerated in opposite directions. They concluded that their method reproduces the transition from a laminar to secondary stationary flow, albeit qualitatively. If the Reynolds number is increased and the secondary flow is no longer characterized by an array of stationary vortices, SPAM can determine the transition to fully developed turbulence. However, the authors could not determine the Reynolds number in the unstable flow regimes.

As mentioned earlier, k-flow arises when fluid is subject to an artificial and sinusoidal force. The resultant flow happens to be periodic as well as similar to common shear flows observed in different forms of modeling and simulation techniques. A number of researchers and mathematicians have studied multidimensional simulations of k-flows. Firstly, the flow was analyzed in two-dimensional numerical simulations. Shebalin and Woodruff performed a three-dimensional simulation of the flow using different forms of viscous stress [84]. Moreover, three-dimensional simulations using the measures of hyperviscosity were carried out by Borue and Orszag [85]. Now the question about alternative grid size dependencies using the Smagorinsky model can be approached using two different approaches and methods. Firstly, a detailed prior analysis is to be performed using direct numerical simulation (DNS) on the available data sets. Later onwards, the resulting velocity field is filtered out using a number of filter widths. For each of these filter widths, the Reynolds stress, as well as the Smagorinsky formula, are evaluated. On the other hand, a second approach can also be used. This method employs the use of the LES experimentation. For the different numerical resolutions, a number of simulations are administered using different or varying values of the Smagorinsky constant [86].

The value that leads to the best resolution helps in determining the grid size dependence. It is important to consider that both of these approaches lead to similar results and conclusions in terms of grid size dependence. An intermediate range of wave numbers has also been reported for grid size dependencies. 
It is imperative to have an enhanced grid size dependence where the low wave numbers are observed. At the same time, satisfactory and reliable results have also been achieved using low resolutions, especially when an appropriate grid size was used. However, a deterioration was seen in terms of the different predictions as well as in the turbulent shear stress. A number of papers and studies confirm the idea of alternating the grid size dependence in order to offer a compensation for all forms of limited under resolution, but still a lot more needs to be done in order to reduce the problems and complexities encountered in simulation and modeling techniques. The goal can be achieved more efficiently and effectively using a specific Smagorinsky constant that can provide the best results. The most appropriate value of the Smagorinsky constant may be assessed by a number of sophisticated approaches. Moreover, another major trend in this regard is measuring and assessing the usefulness of the LES [86].

The results of the study conducted by Woodruff Seiner et al. [87] indicate that most of the low-resolution simulations are able to reproduce the resulting kinetic energy. Furthermore, these simulations are also able to reproduce almost all of the statistical quantities. However, the low-level resolutions failed to determine the correlation coefficient $C x z$. It is evident that the low-resolution simulations are the most recommended under similar circumstances in order to provide for optimal and reliable results. The results also reported no form of sensitivity in terms of the correlation coefficient and the changing values of the Smagorinsky constant. Thus, it is clear that the simulations that were performed using the Smagorinsky constant failed to determine the exact value of the correlation coefficient. For this reason, it is clear that no simple tuning method of the constant is good enough to determine the correct value for the correlation coefficient. In simple words, it can be said that a more drastic adjustment and restructuring of the model is needed for more appropriate measurements and calculations of the coefficient [86].

Simulation of turbulent flows is also attracting a great deal of attention these days. Modeling and simulation of k-flow can be analyzed through large eddy simulations (LES). The core idea behind any form of large eddy simulation is that almost all of the largest turbulent scales can be resolved in a numerical manner. At the same time, it also asserts that only the smallest, as well as self-similar scales, might be helpful in the modeling process. If one could resolve all scales in terms of large and small scales of the inertial range, the developed model would be relatively basic and effectively computable. One such model is the Smagorinsky model that, despite its faults and drawbacks, has been used successfully until now for carrying out large eddy simulations. However, it is a reality that this concept can be used with complete accuracy for the simplest models. For the more complex models, the available computational techniques and resources only allow for a resolution into two transitional regions: the inertial and energy containing ranges [87].

The complex and fastest computational machines will be able to make a small dent in the problem. We can use these computational devices and methodologies 
for the solution of complex flows. An important question in this regard is to ask what might be the consequences and outcomes of making use of these inadequate resolution methods and what measures should be taken for their amelioration. It is also evident that there is a need to look for alternative and more effective simulation and modeling techniques. It is also important to ask whether an alternative non-grid size dependence and measure be used for improving LES of k-flow. The possibility of having different alternatives in terms of grid size dependencies has been raised by a number of researchers and experts. With this said, it is possible to make use of alternative grid size dependencies of the Smagorinsky model in relevance of the LES of k-flow [87].

In addition to having a number of computational and numerical advantages, the k-flow also offers a mechanism for testing different turbulence and simulation models. Using this approach, almost all of the turbulent flow features can be assessed and measured in a detailed and effective manner. A number of approaches have been proposed for the development and formulation of time-dependent models and techniques in relation to non-equilibrium flows [86]. This analysis of the different modeling and simulation techniques yielded two primary questions. Firstly, under what conditions and circumstances a steady-state model can be used for assessing the non-equilibrium turbulent flows. The second question is whether or not the simulation and modeling techniques specified above can help in improving the drawbacks and de-merits of the Smagorinsky model. It has also been observed that the agreement between the LES measures and DNS techniques decreases with the increase in the wave numbers of the forcing. Thus, it is now evident that Smagorinsky model and all other modeling techniques lag behind in terms of generating satisfactory results for the non-equilibrium turbulent flows.

Mainly due to the explosive rate at which computing power increased, most of the numerical simulation studies took place during the last decade. The exponentially increasing processing power of modern computers was also the herald of more detailed, complex numerical models. In order to formulate more detailed and comprehensive models of turbulence, there is a need for performing accurate predictions and estimates of the dissipation factor. Furthermore, the values of the Reynolds numbers will also need to be evaluated in this regard. The study of turbulence driven by a number of Kolmogorov forces using different single number profiles was conducted by Borue \& Orszag [85]. Since then, a number of attempts have been made for understanding the force shape dependence in terms of turbulence [9].

By using direct numerical simulations, Schaefer et al. [88] tested the model equations for the mean dissipation using a k-flow. The authors compared the standard model [89] and a transformed Menter's $k-\varphi$ model [90], which included a cross diffusion and second production terms, against the results of direct numerical simulations. Due to the second production term, the transformed model displayed superior behavior than the standard model; however, the cross-diffusion term held no importance for obtaining a steady solution. Zhang 
and Fan [91] simulated two-dimensional k-flow via the direct Monte Carlo method. Their simulations have been performed for a Knudsen number of 0.005 and the authors observed two main regimes, with each of them corresponding to different ranges of the Reynolds number. The results of their simulations were consistent with those obtained by solving the incompressible viscous Navier-Stokes equations.

Sarris et al. [92] studied the Kolmogorov flow generated by a stationary one-dimensional forcing varying sinusoidally in space using direct numerical simulations with periodic boundary conditions. The study aimed to display the effect that computational box size has on the calculation of the properties of the Kolmogorov flow. The study concluded that turbulence statistics are heavily dependent on the boundary conditions that have been chosen, observing that some symmetries compatible with both the boundary condition and the forcing are broken in the statistical sense. The authors suggested that Kolmogorov flow could thus be considered as an appropriate test case for assessing large-eddy simulation of inhomogeneous, anisotropic, and sheared turbulent flow, without having to deal with the problem of wall modeling.

The energy-enstrophy method, a nonlinear stability method, was introduced in 2008 by Tsang and Young [93]. The proposed method was specialized for two-dimensional hydrodynamics and developed from a nonlinear stability analysis of the k-flow. The study was focused on the limit in which drag is much stronger than viscosity, motivated by the possibility of applying the technique of Doering and Constantin [10] [94] to two-dimensional turbulence, where it is essential to take into account the enstrophy conservation. In an attempt to simplify the great computing power required to model large complex flow systems, Kramar et al. [95] presented an analysis of Kolmogorov flow and Rayleigh-Benard convection using persistent homology as a data reduction method. Two Kolmogorov flow regimes are studied: chaotic dynamics from the appearance of unstable fixed point and a periodic flow that displays drift in the direction of symmetry. The authors took a general approach in order to maximize the applicability of their method on open problems that exhibit complex spatiotemporal behavior. According to the results of the study, persistent homology is an effective method both for quotienting out symmetries in families of solutions and for identifying multiscale recurrent dynamics. The authors concluded that persistent homology is a method robust to noise and sensitive to complicated dynamics, appropriate for studying experimentally acquired data sets.

\subsection{Experimental Studies}

\subsubsection{Experimental Study of Kolmogorov Flows Using Cylindrical Surface}

In this experiment, a laboratory model of the k-flow was investigated using a cylindrical surface. The different number of half periods in terms of the external force was varied from 2 to 22 . It was shown that the specific type of secondary flows is determined and dependent on the number of half periods in relation to 
the basic flow. The half periods include the traveling wave for all of the odd number of periods, a self-oscillating regime and a quasi-steady vortex structure. This form of theoretical analysis has been based on the Galerkin approximation. Moreover, the system of equations that were obtained was solved numerically in direct conjunction with the analysis [96]. The different experiments that have been carried out in the laboratory settings have shown that there is a specific interval marked for its super-criticality in relevance to the secondary flows. One reason can be the friction of the fluid generated with the channel bottom due to which the stability curve is modified. The confinement of the observed flow with the sidewalls also leads to similar results [90]. The results of the study showed that some of the most dangerous disturbances are marked for having wave numbers close to 0.31 . It is an indication that the number of vortices formed alongside the $\mathrm{x}$-axis happen to be independent of the channel width. At the same time, the nature of the fluid motion in terms of the super critical regime is determined based on the imaginary part. In terms of the supercritical regime, three different solutions were observed. It was also reported that the behavior of the observed flow in the supercritical regime was confirmed with the numerical integration of the system being investigated [96].

An experimental apparatus with mechanical periodic (but not sinusoidal) forcing has been presented, which the authors used to investigate the instability of k-flows in a soap film [42]. The results of the experimental study were used for comparison against the numerical models of older studies, which displayed virtually no convergence with the experimental results.

For the purpose of an undergraduate laboratory experiment, Kelley and Ouellette have constructed an apparatus to create a quasi-two-dimensional flow, using electromagnetically driven thin-layer flow [97]. The authors noted that this approach has been selected over soap films because of its simplicity, as the students will be called to setup the experiment. The paper summarizes the most important, basic theory regarding the k-flow, places focus on the experimental setup and data acquisition procedures and, finally, discusses the pedagogical aspects of the project.

By using the experimental setup suggested by Rivera and Ecke [98], Suri et al. [21] investigated the velocity profiles in two-dimensional k-flow. The authors confined their comparisons between theoretical and experimental results to the laminar flow because, as they proclaim, they sought closed form expressions for the coefficients in the $2 \mathrm{D}$ vorticity equation, in order to gain insight into how they depend on various experimental parameters. Within these parameters, their study displayed excellent agreement between experimental measurements and analytical predictions, while the authors also concluded that increasing the viscosity of the electrolyte relative to that of the dielectric would improve the uniformity of the flow.

\subsubsection{Lamination and Mixing in Laminar Flows Using Lorentz Body Forces}

In this experimental investigation, a relatively newer approach was demonstrat- 
ed for the designing of different mixtures. The approach is centered on using a sequence of different tailored flows in combination with a new procedure for quantifying varying levels of striation. The process, referred to as lamination, can be seen as translating to the specific distance over which different forms of molecular diffusion will need to act. In situ, the process of mixing was also achieved using a tailored sequencing of different flows. The degree of mixing that was observed showed an exponential growth before the saturation was achieved. This form of saturation is seen when the thickness of the striations happens to be smaller than the length scale [99].

It should be noted that without the molecular diffusion, the thickness of the striations would have been smaller in comparison to the size of the atom. The results of the study showed that 3 minutes are enough for mixing the species with low levels of diffusivities. Moreover, the stretching, as well as the lamination, showed an exponential growth. For each of the forcing periods, the lengths of the line in combination to its lamination were multiplied with 23. After a timing gap of three minutes, the average lamination was reported to be 3000 with the striation thickness being about $3.3 \mu \mathrm{m}$. It was also shown that the in situ mixer did not demand a mean flow inside the pipe for efficient mixing. The primary mechanism for the mixing process was the control of two local jets. It should also be considered that such flows can be designed using a number of other devices. The study also pointed out the need of using green mixing so that it might consume lower amounts of energy [99].

\subsubsection{Numerical and Experimental Study of a Circular Shear Layer}

The experiment by Chomaz et al. [100] showed that the dynamical behavior of the observed flow was dependent on the aspect ratios of the cell. In terms of the larger cells, the transition from the mode having a lower number of vortices towards a mode with higher number of vortices is determined using a number of localized processes. The transition is seen to occur after a series of different bifurcations that happen to be in correspondence with the successive breaking of the different symmetries of the flow being studied. The results of the study showed that a two-dimensional simulation of the flow is sufficient for recovering the varying dynamical processes of the experimental flow. The rational variance was visible during the different phases of the experiment. In the experiment, there was no sort of translational variance reported. It was also shown that for the different velocities, a number of specific forces could be easily neglected. It was due to this that the rotating frames were undistinguishable just like the Galilean frames. A distinction between the convective and absolute instabilities was also observed. The case is important for having a spatially periodic flow as reported by the researchers who performed the experiment. The numerical simulations observed also happened to be similar to the simulation of linear shear flows. The researchers reported that the experiment was seen to be lagging in were some dimensions and domains. However, the study managed to provide a number of new insights about the experimental investigations of k-flow [100]. 


\subsubsection{An analysis of Forced Periodic Flows and Their Spatio-Temporal Dynamics}

The work reported in [99] showed that spatiotemporal chaos could be caused due to the competition between the varying unstable modes. The instabilities and dynamics of a specific localized vortex were analyzed using different experimental procedures. A double bifurcation was seen in addition to a new periodic state. The results showed that the instability threshold was in close accordance with the experimental one. The findings of this experiment were in close relevance with the previous studies and investigations that were carried out in this regard. Moreover, this study pointed out to the need of more future studies for evaluating the forced periodic flows in more detail [101].

\subsubsection{Experimental Investigation of the Quasi-Two Dimensional Shear Flows}

In the experimental study reported in [100], forced shear flows were investigated in a thin layer using an in-viscous fluid. In order to obtain the stream function of the observed vertical flow, a number of streak photographs were taken. Different flow characteristics were determined by investigating these flows. For the purpose of evaluation, the experimental flow was observed using a MHD apparatus. Moreover, a magnetic field was also created using circular magnets. In order to generate a shear flow, a number of cylindrical electrodes were utilized. The Kolmogorov flow was generated using a number of different devices and instruments [102].

The mean velocity, vorticity and Reynolds stress were also measured in [100] and a harmonic analysis of the resulting disturbances was performed keeping in view the dynamics of the system. The conclusions of the experiment showed a verification of quasi-two-dimensional approximation for thin-layered fluids. The results also gave an indication about the applicability of Q2D approximations. At the same time, the possibility of reconstructing Q2D shear motions was also investigated. Correct behavior was determined for both of the profiles being investigated. The force profiles were analyzed in relevance to these approximations. Another important result of the experiment was that Q2D flows could also be applied to the varying atmospheric flows. However, it should be noted that the specific method being proposed here could not be applied directly to the atmosphere, with the main reason being the need for a complete resolution of the observed vertical structure. Keeping in view the data obtained from the horizontal fields at varying altitude levels, this specific procedure can be generalized for different forms of reconstruction using the vorticity transformation equation reported in [102].

\subsubsection{Turbulence of Shallow Water Flows Modeling}

The most recent modeling study using the Kolmogorov approach was performed by $\mathrm{Pu}$ [103], who explored the turbulence of shallow water flows. To that end, the author combined a model of shallow water equations with Kolmogorov's $k-$ $E$ turbulence model and verified the simulations by comparing them to experi- 
mental data. He also compared the results of both the newly developed model and the validation experiments to previous studies, mainly focusing on comparisons with the Boussinesq model [104]. According to the author's conclusion, the newly developed model reproduced the flow characteristics of multiple-obstructions induced flow reasonably well. The author also notes that the Kolmogorov scaling model should be given more attention by future studies as an achievable approach to resolve computationally demanding flow turbulence.

\subsubsection{Simulations on Turbulent Kolmogorov Flow without Boundaries} Musacchio and Boffetta published the results of numerical simulations of turbulent Kolmogorov flow without boundaries [105]. The main aim of the study was to examine the dependence of turbulent drag on the Reynolds number, but the researchers also presented a detailed analysis of the scale-by-scale energy balance that shows how the kinetic energy is redistributed among different regions and scales. The study derives a prediction for the spatial transport of kinetic energy, describing how it is redistributed among different regions of the flow. The authors conclude that the Kolmogorov flow is the ideal framework to investigate the properties of spatial transfer of kinetic energy in nonhomogeneous, turbulent sheared flows.

\subsubsection{Spatiotemporal Dynamics in Two-Dimensional Kolmogorov Flow} Lucas and Kerswell [106] studied the spatiotemporal dynamics in two-dimensional Kolmogorov flow over large domains. The numerical study was aiming to examine the 2D Kolmogorov flow over an extended domain that would display spatially localized chaotic flows, i.e. points that would approach 2D turbulence. The results displayed rich spatiotemporal behavior once larger domains are considered, focusing on the existence of localized flow structures. However, the authors conclude that the disparity between the large domains used for the means of their study and the spatial extent of the localized chaos that exists is a major challenge, requiring the development of more efficient recurrent flow analysis strategies.

\subsubsection{Transition to Turbulence in the Three-Dimensional Kolmogorov Flow}

One of the few studies that examine three-dimensional Kolmogorov flows is the recently published paper of Veen and Goto [107], in which they examine the transition from a three-dimensional Kolmogorov flow to turbulence via numerical simulations. The authors study the subcritical transition process assuming the "simplest possible circumstances" of a flow on a triply periodic domain with aspect ratios equal to unity and forcing with the smallest wave number in one direction only. Their work reveals the presence of an equilibrium state close to the laminar flow with no drift in the streamwise or spanwise directions.

\section{Conclusions}

The research works of Andrei Nikolai Kolmogorov have aided science in terms 
of getting the answers and solutions for some of the most perplexing phenomena including turbulence, shear flows, fluidic behaviors, and probabilities. The study of two-dimensional flows that was initiated by Kolmogorov was continued by future researchers and mathematicians, and the analysis of magnetohydrodynamics and the mathematics behind them clearly indicate that the Kolmogorov flows (k-flows) are a major subject of investigation. Numerical simulations and investigations have helped a lot in advancing our understanding of these flows. As specified in this review paper, it is evident that a number of efforts have been made in terms of understanding the laboratory measurements and realizations of these flows. The contributions of Kolmogorov in the field of fluid dynamics cannot be undermined at any level. It was due to this works that we are now able to better understand the velocity fields in a number of intermediate scales, chaotic flows, and the inertial shear range. In addition, his 5/3 law has also remained a major landmark in this field, which was used by a number of researchers and scientists have put forward revolutionary theories on fluid dynamics.

It can be concluded that the study of these flows has helped in assessing the stability of the viscous shear flows and the different behaviors that are exhibited by fluids. At the same time, the phenomenon of turbulence has been studied in detail using the principles and concepts put forward by Kolmogorov. It is also evident that we are still too far from grasping a number of randomized and chaotic behaviors that are exhibited by fluids. The Reynolds number has also been critical in terms of these investigations. All the studies and researchers cited above used different values of the Reynolds number in order to gain a deeper understanding of the stability and instability of k-flows. The studies have explored whether the different small-scale forces, such as the baroclinic instability cascade, combine to form different planetary flows. Thus, it can be said that these studies have described two physical effects that may be asserted as applications of k-flows. These two effects are the beta effect and bottom friction. The analysis has also reported that vorticity and turbulence have remained a central topic in the domain of magnetohydrodynamics.

Kolmogorov was able to present his concepts and studies in a clear way. The dimensional analyses he performed are still being used as the basis for a number of experimental studies. However, there are some weak points that cannot be ignored. Despite the total success of the theory in terms of describing a number of crude features of fully developed turbulence, a number of facets and aspects of his propositions do not fit well with the actual turbulence. Turbulence is indeed a complex phenomenon due to which it is difficult to entirely access and evaluate its nature. Moreover, it is indeed a difficult challenge to formulate a fully-fledged theory of turbulence without any sort of limitation. There are always limitations even in some of the best and most comprehensive theories known.

Future research and studies should be dedicated towards the applications of the concepts and studies of Kolmogorov for investigating new domains and frontiers. In other words, the focus of the researchers and mathematicians 
should be to use Kolmogorov's works in something that will help science. The applications of k-flows in the domain of engineering and all other related fields are numerous due to which special attention should be paid to understanding them in detail. The contributions of Kolmogorov are not limited to fluid dynamics, mechanics, magnetohydrodynamics and mathematics. It was one of his major interests to apply statistical theories and principles to real life settings. For those who do not know, k-flow has also been extensively applied in the field of economics [108], biology [109] and even data encryption [110]. More specifically, some studies are concerned with the applications of his equations in the simulation of financial activities. In simple words, his works have been applied in financial simulation modeling. His equations and works have been used for formalizing comprehensive mathematical models. The behavior of turbulent eddies is now more thoroughly understood, the credit for which directly goes to the works of Kolmogorov. Modern methods and techniques of computerized computational are also somewhat based on the insights and solutions that were derived from the works of Kolmogorov. Whether it is turbulence, heat transfer, stability of shear flows, bifurcation, stratification or simulation, the applications of $k$-flow can be seen in all these domains.

As a recommendation, it is suggested that future studies should be dedicated to limiting the intricacies and complexities of turbulence and shear flows. These studies should not only focus on understanding the theory put forward by Kolmogorov, but also on its applications in different fields. In addition, it is also right to say that more detailed and perfect turbulence models can be formulated using the work of Kolmogorov. Scientists and mathematicians have been interested in applying the concepts of turbulence to complex flows. That is not all, as Kolmogorov's ideas have also been used in the formulation of algebraic turbulence models. In short, the field of magnetohydrodynamics can excel through understanding and applying the works and studies of Kolmogorov.

\section{Conflicts of Interest}

The author declares no conflicts of interest regarding the publication of this paper.

\section{References}

[1] Balmforth, N.J. and Young, Y.-N. (2002) Stratified Kolmogorov Flow. Journal of Fluid Mechanics, 450, 131-167. https://doi.org/10.1017/S0022111002006371

[2] Balmforth, N.J. and Young, Y.-N. (2005) Stratified Kolmogorov Flow. Part 2. Journal of Fluid Mechanics, 528, 23-42. https://doi.org/10.1017/S002211200400271X

[3] Young, Y.-N. and Balmforth, N. (2000) On Stratified Kolmogorov Flow. Stirring and Mixing: 1999 Program of Summer Study in Geophysical Fluid Dynamics, 97.

[4] Obukhov, A. (1983) Kolmogorov Flow and Laboratory Simulation of It. Russian Mathematical Surveys, 38, 113-126. https://doi.org/10.1070/RM1983v038n04ABEH004207

[5] Meshalkin, L. and Sinai, I.G. (1961) Investigation of the Stability of a Stationary So- 
lution of a System of Equations for the Plane Movement of an Incompressible Viscous Liquid. Journal of Applied Mathematics and Mechanics, 25, 1700-1705. https://doi.org/10.1016/0021-8928(62)90149-1

[6] Kolmogorov, A.N. (1941) The Local Structure of Turbulence in Incompressible Viscous Fluid for Very Large Reynolds Numbers. Doklady Akademii Nauk SSSR, 299-303.

[7] Carbone, V., Veltri, P. and Bruno, R. (1995) Experimental Evidence for Differences in the Extended Self-Similarity Scaling Laws between Fluid and Magnetohydrodynamic Turbulent Flows. Physical Review Letters, 75, 3110. https://doi.org/10.1103/PhysRevLett.75.3110

[8] Richardson, L.F. (1922) Forms Whereon to Write the Numerical Calculations Described in Weather Prediction by Numerical Process. Cambridge University Press, Cambridge.

[9] Rollin, B., Dubief, Y. and Doering, C. (2011) Variations on Kolmogorov Flow: Turbulent Energy Dissipation and Mean Flow Profiles. Journal of Fluid Mechanics, 670, 204-213. https://doi.org/10.1017/S0022112010006294

[10] Doering, C.R. and Constantin, P. (1992) Energy Dissipation in Shear Driven Turbulence. Physical Review Letters, 69, 3000. https://doi.org/10.1103/PhysRevLett.69.3000.2

[11] Pope, S.B. (2000) Turbulent Flows. Cambridge University Press, Cambridge, 806. https://doi.org/10.1017/CBO9780511840531

[12] Weinan, E. and Shu, C.W. (1993) Effective Equations and the Inverse Cascade Theory for Kolmogorov Flows. Physics of Fluids A: Fluid Dynamics (1989-1993), 5, 998-1010. https://doi.org/10.1063/1.858644

[13] Kraichnan, R.H. (1959) The Structure of Isotropic Turbulence at Very High Reynolds Numbers. Journal of Fluid Mechanics, 5, 497-543. https://doi.org/10.1017/S0022112059000362

[14] Kraichnan, R.H. (1965) Inertial-Range Spectrum of Hydromagnetic Turbulence. Physics of Fluids (US), 8, 1385. https://doi.org/10.1063/1.1761412

[15] Kraichnan, R.H. (1976) Eddy Viscosity in Two and Three Dimensions. Journal of the Atmospheric Sciences, 33, 1521-1536. https://doi.org/10.1175/1520-0469(1976)033<1521:EVITAT>2.0.CO;2

[16] Ecke, R. (2005) The Turbulence Problem. An Experimentalist's Perspective. Los Alamos Science, Los Alamos.

[17] Hunt, J.C., Phillips, O.M. and Williams, D. (1991) Turbulence and Stochastic Processes: Kolmogorov's Ideas 50 Years On. Royal Society, London.

[18] Frisch, U. (1991) From Global Scaling, a la Kolmogorov, to Local Multifractal Scaling in Fully Developed Turbulence. Proceedings of the Royal Society of London. Series A: Mathematical and Physical Sciences, 434, 89-99. https://doi.org/10.1098/rspa.1991.0082

[19] Hunt, J. and Vassilicos, J. (1991) Kolmogorov's Contributions to the Physical and Geometrical Understanding of Small-Scale Turbulence and Recent Developments. Proceedings of the Royal Society of London A: Mathematical, Physical and Engineering Sciences, 434, 183-210. https://doi.org/10.1098/rspa.1991.0088

[20] Bray, K.N.C. and Cant, R.S. (1991) Some Applications of Kolmogorov's Turbulence Research in the Field of Combustion. Proceedings of the Royal Society of London. Series A: Mathematical and Physical Sciences, 434, 217-240.

https://doi.org/10.1098/rspa.1991.0090 
[21] Suri, B., Tithof, J., Mitchell, R., Grigoriev, R.O. and Schatz, M.F. (2014) Velocity Profile in a Two-Layer Kolmogorov-Like Flow. Physics of Fluids (1994-Present), 26, Article ID: 053601. https://doi.org/10.1063/1.4873417

[22] Boratav, O.N. and Pelz, R.B. (1997) Structures and Structure Functions in the Inertial Range of Turbulence. Physics of Fluids (1994-Present), 9, 1400-1415. https://doi.org/10.1063/1.869253

[23] Lilly, D. (1971) Numerical Simulation of Developing and Decaying Two-Dimensional Turbulence. Journal of Fluid Mechanics, 45, 395-415. https://doi.org/10.1017/S0022112071000107

[24] Fortova, S. (2013) Numerical Simulation of the Three-Dimensional Kolmogorov Flow in a Shear Layer. Computational Mathematics and Mathematical Physics, 53, 311-319. https://doi.org/10.1134/S0965542513030056

[25] Kolmogorov, A.N. and Shiryayev, A.N. (1992) Selected Works of an Kolmogorov: Vol. 2, Probability Theory and Mathematical Statistics. Kluwer Academic, Berlin.

[26] Bondarenko, N., Gak, M. and Dolzhanskii, F. (1979) Laboratory and Theoretical Models of Plane Periodic Flow. Akademiia Nauk SSSR Fizika Atmosfery i Okeana, 15, 1017-1026.

[27] Boffetta, G., Celani, A. and Mazzino, A. (2005) Drag Reduction in the Turbulent Kolmogorov Flow. Physical Review E, 71, Article ID: 036307. https://doi.org/10.1103/PhysRevE.71.036307

[28] Brutyan, M. and Krapivskii, P. (1991) Stability of Kolmogorov Flow in a Viscoelastic Fluid. Izvestiya Akademii Nauk SSSR, Mekhanika Zhidkosti, 4, 17.

[29] Thess, A. (1992) Instabilities in Two-Dimensional Spatially Periodic Flows. Part I: Kolmogorov Flow. Physics of Fluids A: Fluid Dynamics (1989-1993), 4, 1385-1395. https://doi.org/10.1063/1.858415

[30] Thess, A. (1992) Instabilities in Two-Dimensional Spatially Periodic Flows. Part II: Square Eddy Lattice. Physics of Fluids A: Fluid Dynamics (1989-1993), 4, 1396-1407. https://doi.org/10.1063/1.858521

[31] Thess, A. (1993) Instabilities in Two-Dimensional Spatially Periodic Flows. Part III: Inviscid Triangular Lattice. Physics of Fluids A: Fluid Dynamics (1989-1993), 5, 335-343. https://doi.org/10.1063/1.858858

[32] Sommeria, J. and Moreau, R. (1982) Why, How, and When, MHD Turbulence Becomes Two-Dimensional. Journal of Fluid Mechanics, 118, 507-518. https://doi.org/10.1017/S0022112082001177

[33] Sommeria, J. (1986) Experimental Study of the Two-Dimensional Inverse Energy Cascade in a Square Box. Journal of Fluid Mechanics, 170, 139-168. https://doi.org/10.1017/S0022112086000836

[34] Thess, A. (1993) Inviscid Instabilities in Two-Dimensional Spatially Periodic Flows. In: Nieuwstadt, F.T.M., Ed., Advances in Turbulence $I V$, Springer Netherlands, 79-84. https://doi.org/10.1007/978-94-011-1689-3_14

[35] Dubrulle, B. and Frisch, U. (1991) Eddy Viscosity of Parity-Invariant Flow. Physical Review A, 43, 5355. https://doi.org/10.1103/PhysRevA.43.5355

[36] Henon, M. and Scholl, H. (1991) Lattice-Gas Simulation of a Nontransverse Large-Scale Instability for a Modified Kolmogorov Flow. Physical Review A, 43, 5365. https://doi.org/10.1103/PhysRevA.43.5365

[37] Frisch, U., Legras, B. and Villone, B. (1996) Large-Scale Kolmogorov Flow on the Beta-Plane and Resonant Wave Interactions. Physica D: Nonlinear Phenomena, 94, 36-56. https://doi.org/10.1016/0167-2789(95)00304-5 
[38] Cahn, J.W. and Hilliard, J.E. (1958) Free Energy of a Nonuniform System. I. Interfacial Free Energy. The Journal of Chemical Physics, 28, 258-267. https://doi.org/10.1063/1.1744102

[39] Wang, L.-P., Chen, S., Brasseur, J.G. and Wyngaard, J.C. (1996) Examination of Hypotheses in the Kolmogorov Refined Turbulence Theory through High-Resolution Simulations. Part 1. Velocity Field. Journal of Fluid Mechanics, 309, 113-156. https://doi.org/10.1017/S0022112096001589

[40] Obukhov, A. (1962) Some Specific Features of Atmospheric Turbulence. Journal of Geophysical Research, 67, 3011-3014. https://doi.org/10.1029/JZ067i008p03011

[41] Wang, L.-P., Chen, S. and Brasseur, J.G. (1999) Examination of Hypotheses in the Kolmogorov Refined Turbulence Theory through High-Resolution Simulations. Part 2. Passive Scalar Field. Journal of Fluid Mechanics, 400, 163-197. https://doi.org/10.1017/S0022112099006448

[42] Burgess, J.M., Bizon, C., McCormick, W., Swift, J. and Swinney, H.L. (1999) Instability of the Kolmogorov Flow in a Soap Film. Physical Review E, 60, 715. https://doi.org/10.1103/PhysRevE.60.715

[43] Childress, S., Kerswell, R. and Gilbert, A. (2001) Bounds on Dissipation for Navier-Stokes Flow with Kolmogorov Forcing. Physica D: Nonlinear Phenomena, 158, 105-128. https://doi.org/10.1016/S0167-2789(01)00320-7

[44] Chen, Z.-M. and Price, W.G. (2002) Supercritical Regimes of Liquid-Metal Fluid Motions in Electromagnetic Fields: Wall-Bounded Flows. Proceedings of the Royal Society of London. Series A: Mathematical, Physical and Engineering Sciences, 458, 2735-2757.

[45] Kolesnikov, Y.B. (1985) Experimental Investigation of Instability of Plane-Parallel Shear Flow in a Magnetic Field. Magnetohydrodynamics, 21, 60-66.

[46] Oparina, E. and Troshkin, O. (2004) Stability of Kolmogorov Flow in a Channel with Rigid Walls. Doklady Physics, 49, 583-587. https://doi.org/10.1134/1.1815419

[47] Chen, Z.-M. and Price, W.G. (2005) Secondary Fluid Flows Driven Electromagnetically in a Two-Dimensional Extended Duct. Proceedings of the Royal Society A: Mathematical, Physical and Engineering Science, 461, 1659-1683.

https://doi.org/10.1098/rspa.2005.1454

[48] Bistagnino, A., Boffetta, G., Celani, A., Mazzino, A., Puliafito, A. and Vergassola, M. (2007) Nonlinear Dynamics of the Viscoelastic Kolmogorov Flow. Journal of Fluid Mechanics, 590, 61-80. https://doi.org/10.1017/S0022112007007859

[49] Oldroyd, J. (1950) On the Formulation of Rheological Equations of State. Proceedings of the Royal Society of London. Series A. Mathematical and Physical Sciences, 200, 523-541. https://doi.org/10.1098/rspa.1950.0035

[50] Berti, S. and Boffetta, G. (2010) Elastic Waves and Transition to Elastic Turbulence in a Two-Dimensional Viscoelastic Kolmogorov Flow. Physical Review E, 82, Article ID: 036314. https://doi.org/10.1103/PhysRevE.82.036314

[51] Mishra, P.K., Herault, J., Fauve, S. and Verma, M.K. (2015) Dynamics of Reversals and Condensates in Two-Dimensional Kolmogorov Flows. Physical Review E, 91, Article ID: 053005. https://doi.org/10.1103/PhysRevE.91.053005

[52] Lorenz, E.N. (1972) Barotropic Instability of Rossby Wave Motion. Journal of the Atmospheric Sciences, 29, 258-265. https://doi.org/10.1175/1520-0469(1972)029<0258:BIORWM>2.0.CO;2

[53] Gill, A. (1974) The Stability of Planetary Waves on an Infinite Beta-Plane. Geophysical and Astrophysical Fluid Dynamics, 6, 29-47. 
https://doi.org/10.1080/03091927409365786

[54] Manfroi, A. and Young, W. (2002) Stability of $\beta$-Plane Kolmogorov Flow. Physica D: Nonlinear Phenomena, 162, 208-232. https://doi.org/10.1016/S0167-2789(01)00384-0

[55] Frenkel, A.L. (1991) Stability of an Oscillating Kolmogorov Flow. Physics of Fluids A: Fluid Dynamics (1989-1993), 3, 1718-1729. https://doi.org/10.1063/1.857951

[56] Feudel, F. and Seehafer, N. (1995) Bifurcations and Pattern Formation in a Two-Dimensional Navier-Stokes Fluid. Physical Review E, 52, 3506. https://doi.org/10.1103/PhysRevE.52.3506

[57] Platt, N., Sirovich, L. and Fitzmaurice, N. (1991) An Investigation of Chaotic Kolmogorov Flows. Physics of Fluids A: Fluid Dynamics (1989-1993), 3, 681-696. https://doi.org/10.1063/1.858074

[58] Love, P. (1999) Bifurcations in Kolmogorov and Taylor-Vortex Flows. Ph.D. Thesis, Center for Research on Parallel Computation, Rice University, Houston.

[59] Legras, B., Villone, B. and Frisch, U. (1999) Dispersive Stabilization of the Inverse Cascade for the Kolmogorov Flow. Physical Review Letters, 82, 4440. https://doi.org/10.1103/PhysRevLett.82.4440

[60] Legras, B. and Villone, B. (2009) Large-Scale Instability of a Generalized Turbulent Kolmogorov Flow. Nonlinear Processes in Geophysics, 16, 569-577. https://doi.org/10.5194/npg-16-569-2009

[61] Matsuda, M. and Miyatake, S. (2002) Bifurcation Analysis of Kolmogorov Flows. Tohoku Mathematical Journal, 54, 329-365. https://doi.org/10.2748/tmj/1113247600

[62] Tithof, J., Suri, B., Pallantla, R.K., Grigoriev, R.O. and Schatz, M.F. (2016) An Experimental and Numerical Investigation of Bifurcations in a Kolmogorov-Like Flow. arXiv Preprint arXiv:1601.00243.

[63] Davis, P. and Peltier, W. (1976) Resonant Parallel Shear Instability in the Stably Stratified Planetary Boundary Layer. Journal of the Atmospheric Sciences, 33, 1287-1300. https://doi.org/10.1175/1520-0469(1976)033<1287:RPSIIT>2.0.CO;2

[64] Sarris, I., Grigoriadis, D. and Vlachos, N. (2010) Laminar Free Convection in a Square Enclosure Driven by the Lorentz Force. Numerical Heat Transfer, Part A: Applications, 58, 923-942. https://doi.org/10.1080/10407782.2010.529034

[65] Holford, J.M. and Linden, P. (1999) Turbulent Mixing in a Stratified Fluid. Dynamics of Atmospheres and Oceans, 30, 173-198. https://doi.org/10.1016/S0377-0265(99)00025-1

[66] Ponetti, G., Sammartino, M. and Sciacca, V. (2016) Transitions in a Stratified Kolmogorov Flow. Ricerche di Matematica, 66, 189-199. https://doi.org/10.1007/s11587-016-0296-6

[67] Linden, P. (1980) Mixing across a Density Interface Produced by Grid Turbulence. Journal of Fluid Mechanics, 100, 691-703. https://doi.org/10.1017/S002211208000136X

[68] Redondo, J., Sanchez, M. and Cantalapiedra, I. (1996) Turbulent Mechanisms in Stratified Fluids. Dynamics of Atmospheres and Oceans, 24, 107-115. https://doi.org/10.1016/0377-0265(95)00454-8

[69] Fernando, H.J. (1991) Turbulent Mixing in Stratified Fluids. Annual Review of Fluid Mechanics, 23, 455-493. https://doi.org/10.1146/annurev.fl.23.010191.002323

[70] Ponetti, G., Sammartino, M. and Sciacca, V. (2014) Formation of Coherent Struc- 
tures in Kolmogorov Flow with Stratification and Drag. Acta Applicandae Mathematicae, 132, 483-492. https://doi.org/10.1007/s10440-014-9927-7

[71] Bena, I., Mansour, M.M. and Baras, F. (1999) Hydrodynamic Fluctuations in the Kolmogorov Flow: Linear Regime. Physical Review E, 59, 5503. https://doi.org/10.1103/PhysRevE.59.5503

[72] Bena, I., Baras, F. and Mansour, M.M. (2000) Hydrodynamic Fluctuations in the Kolmogorov Flow: Nonlinear Regime. Physical Review E, 62, 6560. https://doi.org/10.1103/PhysRevE.62.6560

[73] Landau, L. and Lifshitz, E. (1987) Fluid Mechanics: Volume 6 (Course of Theoretical Physics). 2nd Edition, Pergamon Press, Exeter.

[74] Mansour, M.M., Van den Broeck, C., Bena, I. and Baras, F. (1999) Spurious Diffusion in Particle Simulations of the Kolmogorov Flow. EPL (Europhysics Letters), 47, 8. https://doi.org/10.1209/epl/i1999-00342-y

[75] Roeller, K., Vollmer, J.R. and Herminghaus, S. (2009) Unstable Kolmogorov Flow in Granular Matter. Chaos (Woodbury, NY), 19, 041106-041106.

https://doi.org/10.1063/1.3202616

[76] Beyer, P. and Benkadda, S. (2001) Advection of Passive Particles in the Kolmogorov Flow. Chaos: An Interdisciplinary Journal of Nonlinear Science, 11, 774-779. https://doi.org/10.1063/1.1406538

[77] Mitchell Jr., R. and Grigoriev, R.O. (2012) Instabilities and Mixing in Two-Dimensional Kolmogorov Flow. arXiv Preprint arXiv:1212.2890.

[78] Birta, L.G. and Özmizrak, F.N. (1996) A Knowledge-Based Approach for the Validation of Simulation Models: The Foundation. ACM Transactions on Modeling and Computer Simulation (TOMACS), 6, 76-98. https://doi.org/10.1145/229493.229511

[79] Olenev, N.N. (2011) Modeling and Simulation Techniques. http://www.eolss.net/outlinecomponents/Systems-Analysis-Modeling-Integrated-W orld-Systems.aspx

[80] Samarskii, A.A. and Mikhailov, A.P. (2001) Principles of Mathematical Modelling: Ideas, Methods, Examples. CRC Press, Boca Raton.

[81] Benettin, G., Galgani, L. and Strelcyn, J.-M. (1976) Kolmogorov Entropy and Numerical Experiments. Physical Review A, 14, 2338. https://doi.org/10.1103/PhysRevA.14.2338

[82] Kalis, K. and Kolesnikov, Y. (1988) Numerical Realization of Kolmogorov Flow in a Strong Magnetic Field. Magnetohydrodynamics (English Translation: Plenum Publishing Corproration, United States), 24, 454-459.

[83] Posch, H. and Hoover, W. (1997) Simulation of Two-Dimensional Kolmogorov Flow with Smooth Particle Applied Mechanics. Physica A: Statistical Mechanics and Its Applications, 240, 286-296. https://doi.org/10.1016/S0378-4371(97)00152-0

[84] Shebalin, J.V. and Woodruff, S.L. (1997) Kolmogorov Flow in Three Dimensions. Physics of Fluids (1994-Present), 9, 164-170. https://doi.org/10.1063/1.869159

[85] Borue, V. and Orszag, S.A. (1996) Numerical Study of Three-Dimensional Kolmogorov Flow at High Reynolds Numbers. Journal of Fluid Mechanics, 306, 293-323. https://doi.org/10.1017/S0022112096001310

[86] Woodruff, S., Seiner, J. and Hussaini, M. (1999). Direct Numerical and Large Eddy Simulations of Non Equilibrium Turbulent Kolmogorov Flow. http://ntrs.nasa.gov/archive/nasa/casi.ntrs.nasa.gov/20000014450_2000013292.pdf

[87] Woodruff, S., Seiner, J. and Hussaini, M. (2000) Grid-Size Dependence in the Large-Eddy Simulation of Kolmogorov Flow. AIAA Journal, 38, 600-604. 
https://doi.org/10.2514/2.1030

[88] Schaefer, P., Gampert, M., Goebbert, J.H., Wang, L. and Peters, N. (2010) Testing of Model Equations for the Mean Dissipation Using Kolmogorov Flows. Flow, Turbulence and Combustion, 85, 225-243. https://doi.org/10.1007/s10494-010-9273-4

[89] Jones, W. and Launder, B. (1972) The Prediction of Laminarization with a Two-Equation Model of Turbulence. International Journal of Heat and Mass Transfer, 15, 301-314. https://doi.org/10.1016/0017-9310(72)90076-2

[90] Menter, F., Egorov, Y. and Rusch, D. (2006) Steady and Unsteady Flow Modelling Using the k-SQRT(k).k l-Model. Turbulence, Heat and Mass Transfer, 5, 25-29.

[91] Zhang, J. and Fan, J. (2011) Monte Carlo Simulation of Two-Dimensional Kolmogorov Flow. 27 th International Symposium on Rarefied Gas Dynamics, Pacific Grove, 10-15 July 2010, 378-384.

[92] Sarris, I., Jeanmart, H., Carati, D. and Winckelmans, G. (2007) Box-Size Dependence and Breaking of Translational Invariance in the Velocity Statistics Computed from Three-Dimensional Turbulent Kolmogorov Flows. Physics of Fluids, 19, Article ID: 095101.

[93] Tsang, Y.-K. and Young, W.R. (2008) Energy-Enstrophy Stability of $\beta$-Plane Kolmogorov Flow with Drag. Physics of Fluids (1994-Present), 20, Article ID: 084102.

[94] Doering, C.R. and Constantin, P. (1994) Variational Bounds on Energy Dissipation in Incompressible Flows: Shear Flow. Physical Review E, 49, 4087.

https://doi.org/10.1103/PhysRevE.49.4087

[95] Kramár, M., Levanger, R., Tithof, J., Suri, B., Xu, M., Paul, M., et al. (2016) Analysis of Kolmogorov Flow and Rayleigh-Bénard Convection using Persistent Homology. Physica D: Nonlinear Phenomena, 334, 82-98. https://doi.org/10.1016/j.physd.2016.02.003

[96] Batchaev, A. and Ponomarev, V. (1989) Experimental and Theoretical Investigation of Kolmogorov Flow on a Cylindrical Surface. Fluid Dynamics, 24, 675-680. https://doi.org/10.1007/BF01051717

[97] Kelley, D.H. and Ouellette, N.T. (2011) Using Particle Tracking to Measure Flow Instabilities in an Undergraduate Laboratory Experiment. American Journal of Physics, 79, 267-273. https://doi.org/10.1119/1.3536647

[98] Rivera, M. and Ecke, R. (2005) Pair Dispersion and Doubling Time Statistics in Two-Dimensional Turbulence. Physical Review Letters, 95, 194503. https://doi.org/10.1103/PhysRevLett.95.194503

[99] Rossi, L., Doorly, D. and Kustrin, D. (2012) Lamination and Mixing in Laminar Flows Driven by Lorentz Body Forces. EPL (Europhysics Letters), 97, 14006. https://doi.org/10.1209/0295-5075/97/14006

[100] Chomaz, J., Rabaud, M., Basdevant, C. and Couder, Y. (1988) Experimental and Numerical Investigation of a Forced Circular Shear Layer. Journal of Fluid Mechanics, 187, 115-140. https://doi.org/10.1017/S0022112088000369

[101] Nakamura, Y. (1997) Spatio-Temporal Dynamics of Forced Periodic Flows in a Confined Domain. Physics of Fluids (1994-Present), 9, 3275-3287. https://doi.org/10.1063/1.869442

[102] Dolzhanskii, F., Krymov, V. and Manin, D.Y. (1992) An Advanced Experimental Investigation of Quasi-Two-Dimensional Shear Flow. Journal of Fluid Mechanics, 241, 705-722. https://doi.org/10.1017/S0022112092002209

[103] Pu, J.H. (2015) Turbulence Modelling of Shallow Water Flows Using Kolmogorov Approach. Computers \& Fluids, 115, 66-74. 
https://doi.org/10.1016/j.compfluid.2015.03.010

[104] Launder, B.E. and Spalding, D.B. (1974) The Numerical Computation of Turbulent Flows. Computer Methods in Applied Mechanics and Engineering, 3, 269-289. https://doi.org/10.1016/0045-7825(74)90029-2

[105] Musacchio, S. and Boffetta, G. (2014) Turbulent Channel without Boundaries: The Periodic Kolmogorov Flow. Physical Review E, 89, Article ID: 023004. https://doi.org/10.1103/PhysRevE.89.023004

[106] Lucas, D. and Kerswell, R. (2014) Spatiotemporal Dynamics in Two-Dimensional Kolmogorov Flow over Large Domains. Journal of Fluid Mechanics, 750, 518-554. https://doi.org/10.1017/jfm.2014.270

[107] van Veen, L. and Goto, S. (2016) Sub Critical Transition to Turbulence in Three-Dimensional Kolmogorov Flow. Fluid Dynamics Research, 48, Article ID: 061425. https://doi.org/10.1088/0169-5983/48/6/061425

[108] Maslov, V. (2008) Economic Law of Increase of Kolmogorov Complexity. Transition from Financial Crisis 2008 to the Zero-Order Phase Transition (Social Explosion). arXiv Preprint arXiv:0812.4737.

[109] Brewer, J.W. (1989) The Age-Dependent Eigenfunctions of Certain Kolmogorov Equations of Engineering, Economics, and Biology. Applied Mathematical Modelling, 13, 47-57. https://doi.org/10.1016/0307-904X(89)90197-2

[110] Scharinger, J. (1997) Fast Encryption of Image Data Using Chaotic Kolmogorov Flows. Journal of Electronic Imaging, 7, 318-326. https://doi.org/10.1117/1.482647 